\title{
Validação de recurso pedagógico para adolescentes sobre métodos contraceptivos
}

\author{
Validation of pedagogical resource for adolescents about contraceptive methods \\ Validación de recurso pedagógico para adolescentes sobre métodos anticonceptivos
}

Thayse Gomes de Almeida

ORCID: https://orcid.org/0000-0001-8747-023X Universidade Federal de Alagoas, Brasil E-mail: thaysegalmeida@gmail.com

Eveline Lucena Vasconcelos

ORCID: https://orcid.org/0000-0003-3522-6248 Universidade Federal de Alagoas, Brasil

E-mail: evelinelucena@gmail.com

Ruth França Cizino da Trindade

ORCID: https://orcid.org/0000-0001-9932-6905

Universidade Federal de Alagoas, Brasil

E-mail: ruth_trindade@yahoo.com.br

Raquel Ferreira Lopes

ORCID: https://orcid.org/0000-0002-2061-7038

Centro Universitário Mário Pontes Jucá, Brasil

E-mail: raquelloppes@gmail.com

Marília Vieira Cavalcante

ORCID: https://orcid.org/0000-0003-0516-9019 Universidade Federal de Alagoas, Brasil

E-mail: mariliavcavalcante@ outlook.com

Jéssica da Silva Melo

ORCID: https://orcid.org/0000-0002-4010-810X Universidade Federal de Alagoas, Brasil

E-mail: melo.jessica@outlook.com

Jane Keyla Souza dos Santos Macedo

ORCID: https://orcid.org/0000-0002-5037-097X

Universidade Federal de Alagoas, Brasil

E-mail: jkeyla_souza@hotmail.com

Isadora Pereira Farias

ORCID: https://orcid.org/0000-0001-5771-8492

Universidade Federal de Alagoas, Brasil

E-mail: isadora.pfarias@gmail.com

Ingrid Martins Leite Lúcio

ORCID: https://orcid.org/0000-0003-2738-7527 Universidade Federal de Alagoas, Brasil E-mail: ingridmll@eenf.ufal.br

\begin{abstract}
Resumo
Objetivo: Validar com especialistas um recurso pedagógico no formato de história em quadrinhos direcionado para adolescentes sobre métodos contraceptivos denominado "Métodos Contraceptivos: Sou jovem e ainda não quero gerar uma vida!", quanto ao conteúdo e aparência englobando a clareza, pertinência e representatividade. Métodos: Trata-se de uma pesquisa metodológica, realizada a partir da validação por juízes. A história em quadrinhos foi apreciada por um comitê formado por onze juízes, distribuídos entre professores da educação básica, pesquisadores e profissionais da rede básica de saúde em que responderam a um questionário com nove perguntas na forma de escala Likert, sobre as imagens, os diálogos, conteúdo e uso. Para análise utilizou-se o Índice de Validade de Conteúdo para medir a proporção dos participantes que estão em concordância sobre os painéis ou itens da história em quadrinhos. Resultados: A história em quadrinhos foi considerada, pelos juízes, como muito boa (6) e boa (2), os diálogos e o assunto fáceis de entender (8) e sem dificuldade (8) e o tema muito importante. Porém foi identificado que alguns aspectos necessitam ser revisados e readequados, principalmente quanto à linguagem verbal e conteúdo. Conclusão: Evidencia-se o desenvolvimento de tecnologias da comunicação que possam ser aplicáveis no processo de ensinoaprendizagem e o papel do recurso para promover saúde, desenvolver habilidades e favorecer educação em saúde.
\end{abstract}

Palavras-chave: Adolescente; Anticoncepção; Educação em saúde.

\footnotetext{
Abstract

Objective: Validate with specialists a pedagogical resource in the form of a comic strip aimed at adolescents on contraceptive methods called “Contraceptive Methods: I'm young and I don't want to generate a life yet!", regarding
} 
content and appearance, including clarity, relevance and representativeness. Methods: It is a methodological research, carried out based on validation by judges. The comic was appreciated by a committee formed by eleven judges, distributed among teachers of basic education, researchers and professionals of the basic health network, in which they answered a questionnaire with nine questions in the form of a Likert scale, about images, dialogues , content and usage. For analysis, the Content Validity Index was used to measure the proportion of participants who are in agreement about the panels or comic book items. Results: The comic book was considered by the judges as very good (6) and good (2), the dialogues and the subject easy to understand (8) and without difficulty (8) and the very important theme. However, it was identified that some aspects need to be revised and readjusted, mainly regarding verbal language and content. Conclusion: The development of communication technologies that may be applicable in the teaching-learning process is evidenced and the role of the resource to promote health, develop skills and favor health education.

Keywords: Adolescent; Contraception; Health education.

\section{Resumen}

Objetivo: Validar con profesionales un recurso pedagógico como historia en cuadrinos para los adolescentes sobre métodos anticonceptivos llamado de "Métodos Anticonceptivos: ¡Soy joven y todavía no quiero generar una vida!", cuanto la perspectiva de contenido y apariencia evidenciando la clareza, pertinencia y representatividad. Método: Investigación metodológica, hecha bajo la validación de los jueces. La historia en cuadrinos fue evaluada por un equipo de once jueces entre profesores de educación infantil, investigadores y profesionales de salud, que contestaron a un cuestionario con nueve preguntas en la forma de la escala de Likert, sobre las imágenes, diálogos y contenido que se utilizó. Para el análisis se utilizó el índice de Validez de Contenido para medir la proporción de participantes que están de acuerdo en relación con los ítems que componen la historia en cuadrinos. Resultados: La historia en cuadrinos fue considerada por los jueces, como muy buena (6), buena (2), con los diálogos y tema fácil de se comprender (8) e con tema muy importante (8). Pero se identificó la necesidad de ser cambiado algunos aspectos con relación al lenguaje verbal y al contenido. Conclusión: Evidencia-se el desarrollo de las tecnologías de comunicación que puedan ser aplicables en el proceso de enseno y aprendizaje y la importancia del recurso para la promoción de salud, desarrollar habilidades y favorecer la educación en salud.

Palabras clave: Adolescente; Anticoncepción; Educación en salud.

\section{Introdução}

A adolescência pode ser definida como o período da vida situado entre 12 e 18 anos (Lei Federal n. 8.069/90). Esse período se refere ao crescimento e desenvolvimento biológico, psicológico e social dos seres humanos, de uma forma dinâmica e em curto período de tempo, em que ocorrem mudanças psicofisiológicas e hormonais, que propiciam o início da vida sexual. Caracterizando-se por um período de novas descobertas onde o adolescente expõe-se a comportamentos de risco (Sales et al., 2020).

O exercício da sexualidade acarreta implicações no processo reprodutivo e na própria saúde do adolescente. Alterações comportamentais podem levá-lo a exercer de forma inconsequente sua sexualidade, gerando inúmeros conflitos e interferindo nos seus planos para o futuro, tais como a gravidez precoce, o aborto, as Infecções Sexualmente Transmissíveis, o abandono escolar, a marginalidade, entre outros (Dias et al., 2017).

Em relação à gravidez na adolescência, discutem-se os aspectos que favorecem o aumento desta ocorrência, destacando-se mudanças nos hábitos sexuais dos adolescentes ressaltando seu início precoce, baixa renda e escolaridade, dificuldades ao acesso a programas de saúde sexual e reprodutiva, falta de informação sobre o uso de métodos contraceptivos e ausência de programas efetivos de apoio e acompanhamento do adolescente (Q. Silva, Menegon, Nunes \& R. Silva, 2018).

As razões para o alto índice de gravidez, crescente, especialmente nos países pobres, e Infecções Sexualmente Transmissíveis na adolescência são atribuídos a não utilização de métodos contraceptivos de forma adequada em razão da própria negação do adolescente quanto à possibilidade de engravidar, os encontros casuais e o ato de assumir sua vida sexual ativa, além de pouco conhecimento em relação aos métodos contraceptivos (Silva et al., 2015).

Desse modo, faz-se necessário, ao abordar adolescentes sobre sexualidade e contracepção, envolvê-lo em estratégias de aprendizagem que facilitem a troca de informação e aquisição de saberes, a fim de promover a reflexão sobre a importância de práticas seguras. Acrescenta-se que este desafio deve ser tratado de forma contínua e ampla: multidisciplinar e 
interdisciplinar, envolvendo famílias, escolas, comunidades, instituições que reúnem jovens e, até mesmo, nos locais de lazer, para que os adolescentes possam lidar com esta situação com mais segurança (Beserra, Sousa, Cardoso \& Alves, 2017).

O recurso pedagógico de escolha, validado neste estudo, foi elaborado em forma de História em Quadrinhos, uma vez que, são meios capazes de informar conteúdos e de formar valores de modo sutil, além disso, utiliza os recursos da escrita e as imagens para entreter e informar. As histórias em quadrinhos chamam a atenção do público jovem, porque apresentam uma narrativa relativamente curta e que geralmente costuma ser envolvente, divertida e dinâmica (Rama \& Vergueiro, 2014).

Em virtude de haver a necessidade de se ter um material educativo que consiga atingir o público (adolescentes) de maneira a ensinar e/ou mudar seus conceitos de educação em saúde reprodutiva, é que este manuscrito apresentou a proposta de validação de objeto de aprendizagem, em formato de História em Quadrinhos, com a intenção de colaborar para o ensino e aprendizagem de adolescentes a respeito do conhecimento e uso de métodos contraceptivos. Diante do exposto surgiu como questão norteadora deste estudo: A história em quadrinhos sobre métodos contraceptivos, utilizada neste estudo, é considerada apropriada como um recurso pedagógico para ser testada e futuramente utilizada com adolescentes?

Assim, o objetivo do presente estudo foi validar com especialistas um recurso pedagógico direcionado para adolescentes sobre métodos contraceptivos.

\section{Metodologia}

Trata-se de uma pesquisa metodológica, de abordagem quanti-qualitativa, com foco na validação de tecnologia educativa. A maior parte dos estudos metodológicos é não experimental e frequentemente focado no desenvolvimento, validação e avaliação de ferramentas e métodos de pesquisa (Polit, Beck \& Hungler, 2011).

Segundo Polit, Beck, \& Hungler (2011) a pesquisa metodológica envolve métodos complexos e sofisticados incluindo o uso de modelos com método misto, que se consolida por meio da triangulação criteriosa de dados quantitativos e qualitativos e tem como vantagens: o potencial de complementação, a incrementação e a validade incrementada. Tanto as técnicas quantitativas quanto as qualitativas têm potencialidades e limitações.

Em geral, elas são utilizadas com propósitos distintos. A vantagem fundamental da integração consiste em retirar o melhor de cada uma para responder uma questão específica, maximizando a quantidade de informações incorporadas ao desenho de pesquisa, favorecendo o seu aprimoramento e elevando a qualidade das conclusões do trabalho (Paranhos et al., 2016).

O presente estudo validou, avaliou e aperfeiçoou uma história em quadrinhos a respeito dos métodos contraceptivos, como um recurso pedagógico para ser utilizado com adolescentes.

A validação da aparência e do conteúdo da história em quadrinhos intitulada: "Métodos contraceptivos: sou jovem e ainda não quero gerar uma vida!", foi realizada por meio da apreciação de um comitê formado por 11 juízes. Este número de juízes segue a recomendação de um grupo de pesquisadores que sugere de seis a vinte sujeitos exercendo o papel de juízes (Haynes, Richard \& Kubany, 1995). Integraram esse grupo de juízes professores da educação básica, pesquisadores atuantes na área da saúde sexual e reprodutiva e profissionais da rede básica de saúde.

Como critérios de inclusão, foi exigido a participação de professores da educação básica que lecionam disciplina de ciências do $6^{\mathrm{a}}$ ao $9^{\mathrm{a}}$ ano, e biologia no ensino médio com experiência na temática; pesquisadores que trabalham com tecnologias educativas atuantes nas áreas de educação e/ou saúde sexual e reprodutiva, e profissionais da rede básica de saúde que desenvolvam ações de promoção da saúde sexual e reprodutiva com adolescentes há pelo menos cinco anos. A amostra dos juízes pesquisadores da área foi traçada a partir do currículo lattes de cada um, extraído da Plataforma Lattes do Conselho Nacional de Desenvolvimento Científico e Tecnológico, utilizando filtros para pesquisadores nas grandes áreas da Ciências da 
saúde e Ciências Humanas - área Educação, com as palavras-chave: adolescência, "educação sexual”, sexualidade, contracepção, "tecnologia da educação", validação e "material educativo".

$\mathrm{Na}$ busca do currículo lattes, não foram identificados professores da educação básica e profissionais de saúde, assim sendo a seleção destes juízes foi realizada através de levantamento feito junto a Secretaria de Educação e Secretaria Municipal de Saúde para encontrar profissionais que atendessem aos critérios de inclusão deste estudo.

A coleta de dados foi realizada no período de junho a setembro de 2016. Para o início da coleta, adotou-se um estudo de validação (Lacerda, Magalhães \& Rezende, 2007), distribuindo os seguintes documentos aos juízes do estudo: carta-convite, com informações sobre o título, objetivos e a justificativa do processo de validação; posteriormente ao aceite foi enviado um exemplar da história em quadrinhos, o Termo de Consentimento Livre e Esclarecidos em duas vias e o instrumento de coleta de dados. Foi dado um prazo médio de 30 dias para cada juiz analisar e devolver o instrumento, entretanto, alguns juízes necessitaram de tempo adicional.

O instrumento de coleta de dados utilizado foi criado pelo grupo de pesquisa envolvido no desenvolvimento do projeto "Série Saúde e Sexualidade", sendo testado por professores, mestres e doutores, vinculados à Universidade Federal de Alagoas (UFAL) e a Universidade de São Paulo (USP) e validado numa pesquisa de mestrado da UFAL.

$\mathrm{O}$ instrumento de coleta de dados compõe-se por duas partes: a primeira referente à caracterização dos juízes e a segunda parte o instrumento se debruça sobre a análise da história em quadrinhos por meio de uma escala de Likert dividida em dois (02) domínios. A escala avalia a história em quadrinhos quanto à clareza (domínio 1) e a pertinência ou representatividade (domínio 2), em que o juiz indica seu grau de concordância ou discordância com declarações relativas à atitude que está sendo medida. Desse modo, as declarações foram avaliadas numa escala de 1 a 4. Os itens de concordância receberam valores positivos ou altos (3 ou 4), enquanto as declarações discordantes receberam valores baixos (1 ou 2 ).

Os juízes avaliaram a história como um todo, determinando sua abrangência. Isto é, se cada domínio ou conceito colocado na história foi adequadamente coberto pelo conjunto de itens e se todas as dimensões foram contempladas. Aos juízes foi solicitado/sugerido recomendar a inclusão ou a eliminação de itens no conteúdo da história, bem como em sua aparência.

Com a intenção de preservar o anonimato dos juízes participantes da pesquisa, optou-se por dar-lhes pseudônimos referentes a personagens das obras de um reconhecido cartunista brasileiro (Cebolinha, Denise, Cascão, Marine, Aninha, Dorinha, Maria Cascuda, Mônica, Maria Cebolinha, Magali e Carminha Frufru).

Para a organização, tabulação e análise dos dados utilizou-se inicialmente o Excel 2010. Os instrumentos respondidos foram digitados em uma planilha eletrônica, sendo realizada a dupla digitação e posterior validação dos dados. Os mesmos sofreram tratamento estatístico descritivo. A base de dados utilizada para a extração dos dados foi estruturada para possibilitar sua análise de acordo com o software estatístico utilizado, neste caso o Statistical Package for the Social Sciences 20. As informações foram apresentadas em forma de tabelas, distribuições de frequências, variabilidade. As respostas qualitativas foram agrupadas em quadros conforme características semelhantes presentes nas mesmas, evidenciando as ideias centrais dos discursos dos juízes.

Para analisar a validade de conteúdo e aparência, foi utilizado o Índice de Validade de Conteúdo (IVC), para medir a proporção de participantes que estão em concordância sobre os painéis ou itens da história em quadrinhos, o que permitiu analisar cada um individualmente e também como um todo.

A fórmula para o cálculo:

$$
\mathrm{IVC}=\frac{\text { No de respostas } 3 \text { e } 4}{\text { Total de respostas }}
$$


O Índice de Validade de Conteúdo foi calculado com base em três equações matemáticas: o S-CVI/Ave (média dos índices de validação de conteúdo para todos os índices da escala), S-CVI/UA (proporção de itens de uma escala que atinge escores 3 realmente relevante e 4 muito relevante, por todos os juízes) e o ICVI (validade de conteúdo dos itens individuais). Salienta-se que o índice de validade de conteúdo varia de -1 e 1, e considera-se válido o item cuja concordância entre os juízes seja igual ou maior que 0,80 (Pedreira, Rocha, Santos, Vasconcelos \& Reis, 2016).

Para garantir a confiabilidade do instrumento utilizado nesta pesquisa, foi calculado o Coeficiente Alfa de Cronbach para medir a correlação entre respostas do instrumento através da análise das respostas dadas pelos Juízes, apresentando uma correlação média entre as perguntas. O Coeficiente Alfa de Cronbach é a estratégia mais utilizada para verificar a consistência interna do instrumento no grupo estudado em que os valores são distribuídos numa escala de 0 a 1 , e é considerado válido ao atingir 0,7 (Tibúrcio et al., 2014; Oviedo \& Campo-Arias, 2005).

$$
\alpha=\frac{K}{K-}\left(1-\frac{\Sigma s_{i}^{2}}{s_{\text {soma }}^{2}}\right)
$$

$s_{i}^{2} k$ é o número de itens, $n$ é o número de respondentes.

$s^{2}{ }_{i}$ é a variância dos $n$ escores das pessoas a $i$-ésimo item $(i=1, \ldots, k)$,

$s^{2}$ soma é a variância dos totais $T j(j=1,2, \ldots, n)$.de escores de cada respondente.

As variâncias são calculadas pela fórmula:

$$
s^{2}=\frac{\Sigma(x-\bar{x})^{2}}{n}
$$

Destaca-se que a pesquisa respeitou os princípios éticos propostos na Resolução n ${ }^{\circ} 466 / 12$ do Conselho Nacional de Saúde e este estudo foi aprovado pelo Comitê de Ética em Pesquisa da Universidade Federal de Alagoas (parecer $770.585 / 2014)$.

\section{Resultados}

O instrumento para a coleta de dados foi avaliado quanto a sua confiabilidade pelo coeficiente de alfa de Conbrach. Foi observado que tanto o bloco referente à clareza, quanto ao bloco referente à representatividade obtiveram um coeficiente de alfa de Conbrach com alto nível de confiabilidade, apresentando respectivamente 0,96 e 0,88.

Após avaliação dos dados quantitativos item por item, o recurso pedagógico foi avaliado como um todo atingindo um índice de concordância de 90,90\%, entre os juízes, em que a resposta 4 (quatro) foi a moda entre as respostas (109), portanto, maior que o valor de referência para validação determinado neste estudo, que é de $80 \%$.

O bloco clareza alcançou isoladamente um nível de concordância de $88,18 \%$, em que a resposta 4 (muito claro) foi a moda entre os juízes. Com relação ao bloco representatividade, foi obtido um nível de concordância entre os juízes de 93,38\%, em que, também, a resposta 4 (Extremamente representativo), foi a moda entre os juízes. Constatando-se a validade da história em quadrinhos para ser utilizada como um recurso pedagógico.

Quanto à clareza, foram avaliados 10 (dez) itens pelos juízes, do total de 110 respostas (score), 97 das mesmas foram positivas quando julgadas como claro (48) ou muito claro (49), alcançando um nível de concordância esperado de 88,18\%. A 
maioria dos itens alcançaram isoladamente um índice de concordância superior a 80\%, destacando-se o item referente aos diálogos, que atingiu o nível máximo de concordância de $100 \%$.

Tabela 1 - Distribuição dos escores e percentual de concordância por itens obtidos dos juízes sobre o recurso pedagógico “Métodos Contraceptivos: Sou jovem e ainda não quero gerar uma vida!”, segundo a clareza. Maceió/AL, 2016.

\begin{tabular}{llllll}
\hline \multicolumn{1}{c}{ Clareza } & 1 & 2 & 3 & 4 & Índice de Concordância/Item \% \\
\hline \hline 1. Título & 1 & 0 & 4 & 6 & 90,91 \\
2. Apresentação & 1 & 1 & 4 & 5 & 90,91 \\
3. Objetivo & 0 & 1 & 3 & 7 & 81,82 \\
4. Relação entre as partes & 1 & 1 & 5 & 4 & 90,91 \\
5. Concisão & 0 & 1 & 6 & 4 & 81,82 \\
6. Estrutura textual & 0 & 2 & 4 & 5 & 90,91 \\
7. Ilustrações & 0 & 1 & 5 & 5 & 100 \\
8. Diálogos & 0 & 0 & 6 & 5 & 90,91 \\
9. Harmonia & 0 & 1 & 6 & 4 & 81,82 \\
10. Vocabulário & 0 & 2 & 5 & 4 & 88,18 \\
\hline Subtotal & 3 & 10 & 48 & 49 & 4,55 \\
\hline \hline Percentual por escore & 2,73 & 9,09 & 43,64 & &
\end{tabular}

Legenda: 1 (Não claro) 2 (Pouco claro) 3 (Claro) 4 (Muito Claro). Fonte: Autora (2020).

Quanto ao bloco referente à representatividade, foram julgados 11 itens, do total de 121 respostas (score), 113 foram positivas, sendo 53 julgadas como representativas e 60 julgadas como extremamente representativas, atingindo um nível de concordância de 93,39\%, demonstrando mais uma vez que o recurso pedagógico foi considerado válido. Dos 11 itens, 4 (quatro) atingiram o percentual máximo de $100 \%$, que foram referentes ao título, tema, qualidade do conteúdo e contribuição da história. Além disso nenhum item atingiu o índice de concordância menor que o estabelecido de 0,80. 
Tabela 2 - Distribuição dos escores e percentual de concordância por itens obtidos dos juízes sobre o recurso pedagógico “Métodos Contraceptivos: Sou jovem e ainda não quero gerar uma vida!”, segundo a representatividade. Maceió/AL, 2016.

\section{Representatividade}

1. Título

2. Tema

3. Originalidade

4. Consistência do conteúdo

5. Qualidade do conteúdo

6. Contribuição da história

7. Design pedagógico

8. Ilustrações

9. Diálogos

10. Compreensão da mensagem

11. Vocabulário

$\begin{array}{llll}1 & 2 & 3 & 4\end{array}$

Índice de Concordância/Item \%

\begin{tabular}{lccccc}
\hline Subtotal & 1 & 7 & 53 & 60 & \\
\hline \hline Percentual por escore & 0,83 & 5,79 & 43,80 & 49,59 & 93,39 \\
\hline
\end{tabular}

Legenda: 1 (Não claro) 2 (Pouco claro) 3 (Claro) 4 (Muito Claro). Fonte: Autora (2020).

Os juízes, quando questionados sobre a utilização da História em Quadrinhos, "Métodos Contraceptivos: Sou jovem e ainda não quero gerar uma vida!”, em salas de aula, como recurso pedagógico, a maioria (n=07) respondeu que o utilizaria, três não responderam ao questionamento e uma juíza, apesar de julgar o material como válido, afirmou que não o usaria.

Os participantes foram interrogados sobre como avaliavam a história, quanto ao entendimento dos diálogos, quanto a dificuldade de entendimento do assunto e a importância/relevância do tema; três não responderam a estas perguntas.

Os oito juízes, que responderam a estes questionamentos, consideraram as conversas da história muito boa (6) e boa (2); os diálogos e o assunto fáceis de entender e sem dificuldade (8) e o tema muito importante para os estudantes (8).

Apesar da avaliação positiva que a História em Quadrinhos recebeu dos juízes, quando questionados se alterariam alguma coisa no recurso pedagógico, três juízes afirmaram que não alterariam nada e oito juízes responderam que alterariam. As sugestões de alteração foram apenas direcionadas à linguagem verbal, conteúdo e ilustrações gráficas. Sendo assim, as sugestões foram analisadas, avaliadas e agrupadas de acordo com as ideias principais que surgiram.

Tratando-se do vocabulário, a juíza Denise, pedagoga há 13 anos, sugeriu a utilização de linguagem menos técnica, abrindo espaço para maior utilização de gírias: "Usar linguagem menos técnica, sugiro incluir algumas gírias”. A juíza Maria Cascuda corrobora com a sugestão de Denise: “Achei o vocabulário muito formal, mudar para um diálogo mais próximo da realidade dos adolescentes dos dias de hoje".

Quanto ao conteúdo da história, algumas sugestões direcionadas às práticas do uso de métodos contraceptivos por adolescentes, também foram referidas, principalmente pela juíza Mônica, enfermeira e pesquisadora atuante na área da saúde sexual e reprodutiva há 9 anos. A primeira observação feita por essa juíza, foi em relação ao uso da minipílula e a injeção trimestral por adolescentes antes dos 18 anos: "Na fala da médica, corrigir onde tem dizendo que não pode ser usada por menores de 16 anos, por antes de 18 anos". Como demonstrado na figura abaixo. 
Figura 1 - Página 18 da HQ intitulada: Métodos Contraceptivos: Sou jovem e ainda não quero gerar uma vida!
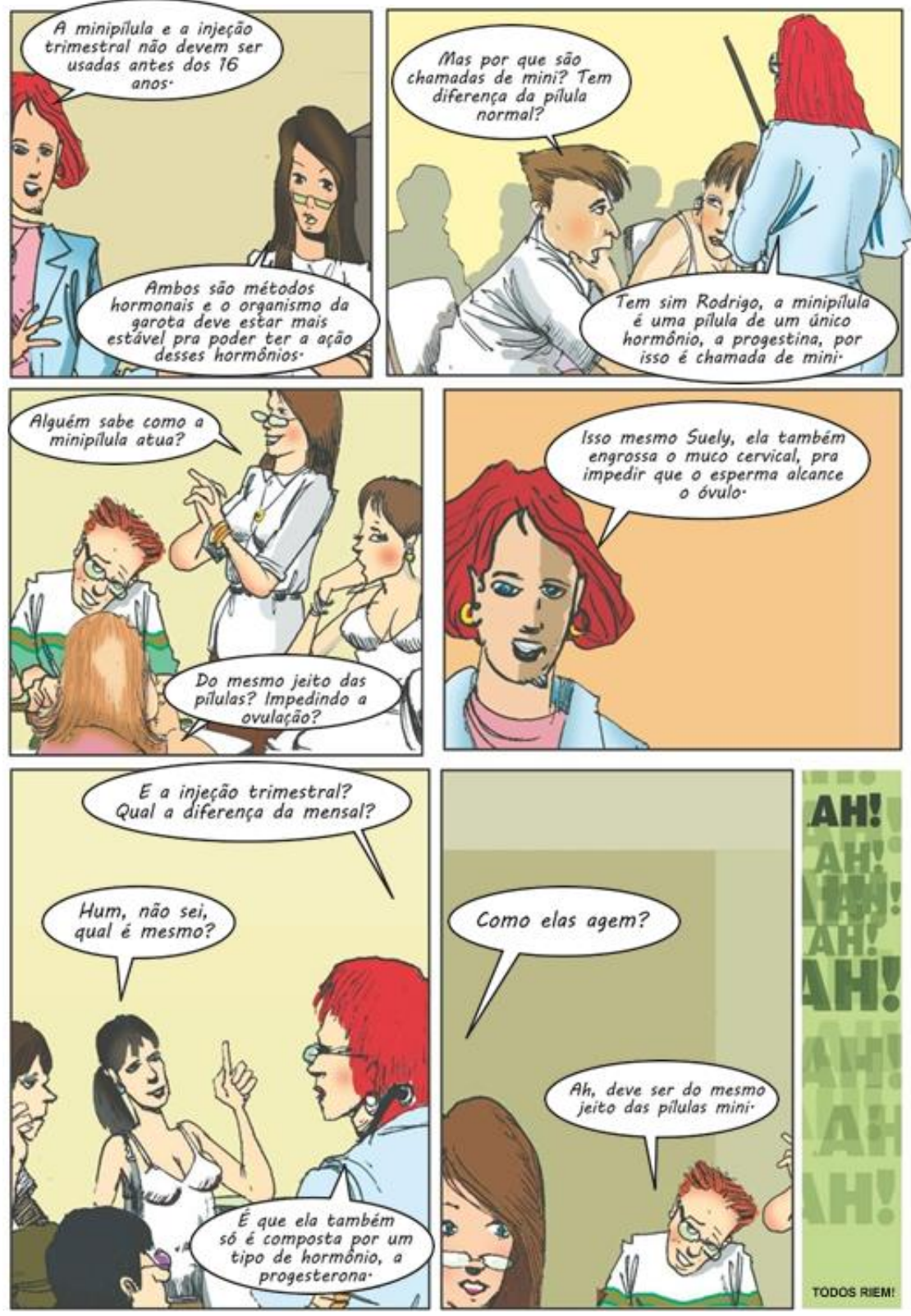

Fonte: Dados da pesquisa (2020).

Em relação ao termo Planejamento familiar, Mônica sugeriu mudança para o termo Planejamento Reprodutivo, justificando que o termo anterior está em desuso: “É necessário fazer atualização: Planejamento familiar por Planejamento reprodutivo".

Segundo a juíza Magali, o termo Doença Sexualmente Transmissível também sofreu mudança, e ela sugeriu que esse termo fosse substituído: "Doença sexualmente transmissíveis por infecções sexualmente transmissíveis".

Em relação ao Dispositivo Intrauterino, Mônica ressalta que a resposta da médica ficou vaga, quando a adolescente questiona quanto a veracidade deste método ser abortivo: "Acrescentar que estudos mais recentes comprovam que o dispositivo intrauterino não é um método abortivo. E o cobre que tem no dispositivo intrauterino é um excelente meio para repelir, afastar os espermatozoides". 
Figura 2 - Página 17 da HQ intitulada: Métodos Contraceptivos: Sou jovem e ainda não quero gerar uma vida!
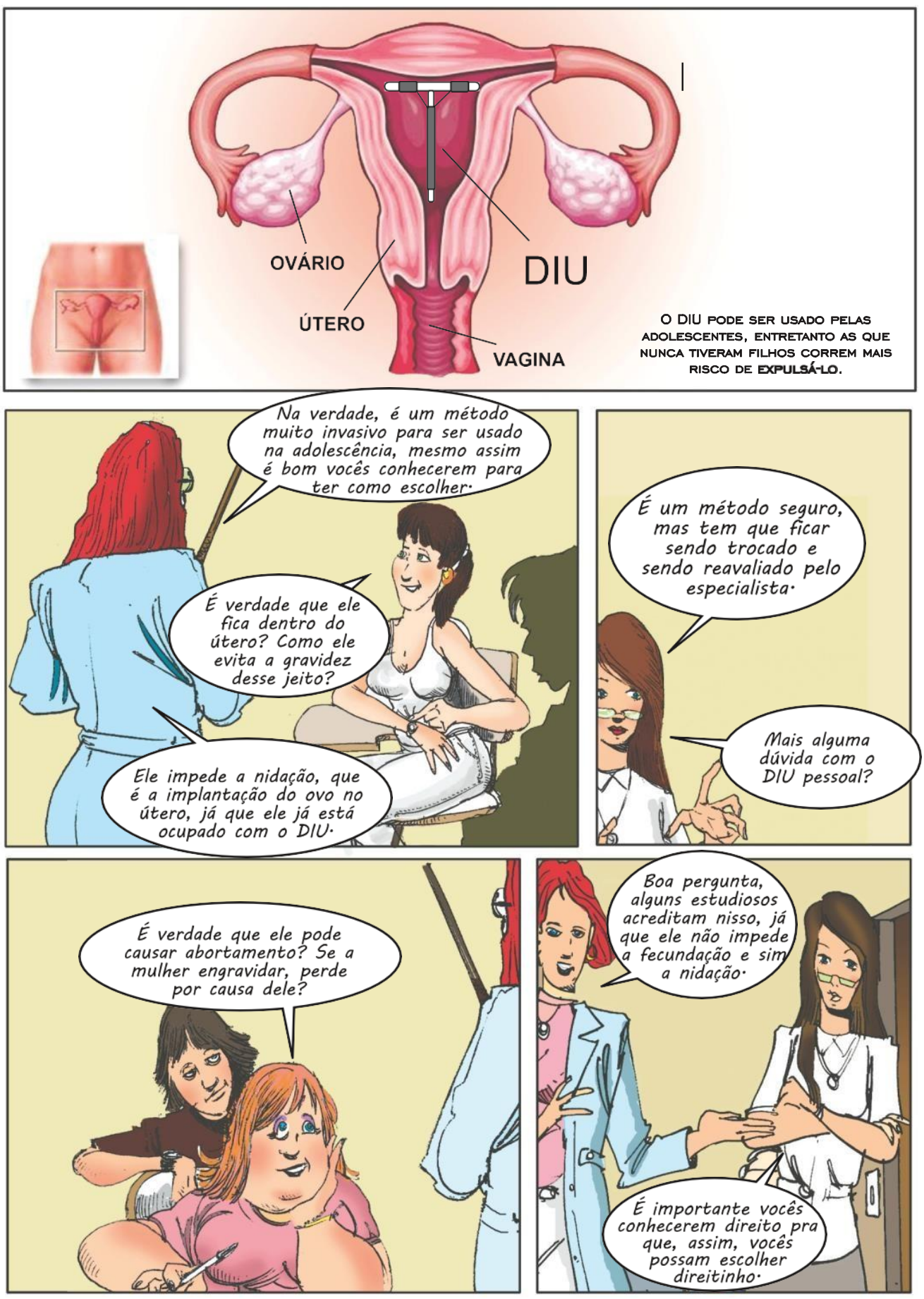

Fonte: Dados da pesquisa (2020).

Por fim, a juíza Dorinha sugere evidenciar a participação da enfermeira na atividade educativa que se passa durante a história: "Acho importante dar mais ênfase na participação da enfermeira nas explicações e na palestra como um todo".

Quanto às ilustrações gráficas foi sugerida por três juízes (Cascão, Marine e Cebolinha) a mudança na aparência dos personagens adolescentes da história em quadrinhos, pois estes demonstravam, de acordo com as ilustrações, falta de interesse pelo tema abordado. 


\section{Discussão}

A inserção das Histórias em Quadrinhos como um recurso pedagógico vem sendo muito discutida. Observa-se que a utilização de histórias em quadrinhos é um poderoso recurso didático/pedagógico por diversos fatores, como estimular o interesse dos estudantes pela leitura, pela praticidade da utilização, e por ser uma nova alternativa de recurso complementar (Santos \& Pereira, 2013). Além disso, se consolida como um instrumento que traz ganhos ao processo de ensinoaprendizagem, pois envolve o estudante, possibilitando que o aprendizado seja diferenciado, contribuindo para a construção de conhecimento, aprimorando a interpretação verbal e visual, facilitando a absorção de conteúdos de maneira lúdica e criativa através do enredo que compõe a história (Amaral \& Locatelli, 2019).

Apesar de desde muito cedo, as histórias em quadrinhos serem objeto de restrição condenadas por muitos pais e professores no mundo inteiro (Rama \& Vergueiro, 2014), isso não foi percebido nas respostas dos juízes, provavelmente, por perceberem que a história em quadrinhos avaliada neste estudo representa um meio de comunicação, cujo conteúdo encontrase direcionado aos jovens.

Nas últimas décadas do século XX, com o desenvolvimento das ciências da comunicação e dos estudos culturais, as histórias em quadrinhos ganharam um novo status, recebendo um pouco mais de atenção das elites intelectuais, sendo aos poucos redescobertas, favorecendo a aproximação das histórias em quadrinhos com as práticas pedagógicas (Rama \& Vergueiro, 2014).

Tratando-se do tipo de linguagem a ser utilizada na história em quadrinhos, esta pode facilitar ou dificultar a captação da mensagem veiculada, devendo ser dispensada atenção especial a esse quesito; a partir da sugestão dada, foi realizada uma revisão geral com o propósito de melhorar e estreitar a comunicação entre o recurso pedagógico e o leitor, visto que, a preparação textual deve estar adequada ao nível educacional e cultural do cliente a ser beneficiado pela tecnologia educativa construída (Teles et al., 2014).

Esta revisão foi realizada de forma cautelosa a pedido de uma das juízas. Observa-se que autores estão buscando nacionalizar, ou mesmo globalizar, suas histórias para que caiam no gosto da sociedade como um todo, independentemente da cultura do leitor. Eles procuram torná-las parte do cotidiano de qualquer cidadão, para que o leitor se sinta familiarizado com a história. Ao valorizarem o cotidiano e ao utilizar em gírias/palavras usadas ou conhecidas pelos alunos nas histórias em quadrinhos, permitem o acesso infanto-juvenil com uma comunicação efetiva (Santos \& Pereira, 2013; Presser \& Schlögl, 2013).

Referindo-se ao uso da minipílula e da injeção trimestral aos 16 anos de idade, a sugestão, da alteração da idade inicial deste, foi discutida entre as pesquisadoras e revisada na literatura. Em 2015 (Finotti, 2015), um estudo mencionou que, de maneira geral, todas as mulheres podem utilizar as minipílulas de forma segura e eficaz, inclusive mulheres que estejam amamentando (iniciando após seis semanas do parto) e em qualquer idade (inclusive adolescentes e mulheres acima de 40 anos). Corroborando com esta premissa outra publicação (Ministério da Saúde, 2016), afirma que o uso da minipílula pode ser feito por toda e qualquer mulher, independentemente se adolescente ou adulta.

Desta forma, optou-se por manter a posição de início, ficando a critério médico a orientação e a adoção ou não deste método contraceptivo, para cada adolescente, durante o planejamento reprodutivo, considerando a singularidade de cada uma.

A literatura traz, ainda, que o termo Planejamento Familiar, está sendo substituído por Planejamento Reprodutivo, visto que, o planejamento pode ser realizado pelo homem e pela mulher, isoladamente, mesmo quando estes não querem instituir uma família. Portanto o assunto já foi e continua sendo bastante discutido, havendo a defesa de que o segundo termo se trata de uma concepção mais abrangente. Pode-se citar como exemplo, o adolescente, o jovem ou o adulto, homem ou mulher, independentemente de ter ou não uma união estável ou de constituir uma família, pode fazer, individualmente ou com 
o(a) parceiro(a), uma escolha quanto a ter ou não ter filhos (Ministério da Saúde, 2016). A partir dessa perspectiva, a sugestão de Mônica foi considerada bastante pertinente e acatada.

A alteração de termos segue através da literatura, também para a terminologia Infecções Sexualmente Transmissíveis, que passou a ser adotada, em substituição à expressão Doenças Sexualmente Transmissíveis, em consonância com a utilização internacional empregada pela Organização Mundial da Saúde, pela Organização Pan-Americana da Saúde, pela sociedade científica e por alguns países. Nesse contexto, alerta-se a população sobre a possibilidade de ter e transmitir uma infecção, mesmo sem sinais e sintomas, o que aponta para estratégias de atenção integral, eficaz e resolutiva (Ministério da Saúde, 2015).

Em relação ao dispositivo intrauterino, que é um dos métodos contraceptivos menos conhecidos pelos adolescentes (Rama \& Vergueiro, 2014), a literatura confirma a fala da juíza, dizendo que a presença de um corpo estranho e de cobre na cavidade endometrial causa mudanças bioquímicas e morfológicas no endométrio, além de produzir modificações no muco cervical. O cobre é responsável pelo aumento da produção de prostaglandinas e pela inibição de enzimas endometriais. Com isso, estas mudanças afetam adversamente o transporte de esperma, de modo a prevenir a fertilização. Os íons de cobre também têm um efeito direto na motilidade espermática, reduzindo a capacidade de penetração no muco cervical (Finotti, 2015). Sendo assim, foi decidido acatar a sugestão da juíza.

Por fim, a sugestão da participação do(a) enfermeiro(a) na atividade educativa foi considerada de grande relevância, visto que, o(a)enfermeiro(a) encontra-se dentre os profissionais que desempenham um importante e necessário papel nas relações entre seres humanos, sociedade, pesquisa, saúde e educação. Uma de suas funções se dá por promover a formação do conhecimento em saúde individual e coletiva, de acordo com a realidade de cada pessoa e grupo social, oportunizando assim a promoção da saúde sob o foco de atitudes (Costa, Figueredo \& Ribeiro, 2013). Ele identifica-se as necessidades e expectativas dos indivíduos pela capacidade de interagir diretamente com o mesmo e a comunidade, bem como pela capacidade de promover o diálogo e interação entre estes e a equipe de saúde (Ribeiro et al., 2016).

A literatura aponta que o(a) enfermeiro(a) possui um papel essencial no desenvolvimento de habilidades preventivas e educativas direcionadas aos adolescentes, com a facilidade de estabelecer estratégias que visam à prevenção da gravidez na adolescência e Infecções Sexualmente Transmissíveis (Ribeiro et al., 2016).

Além disso, o(a)enfermeiro(a) pode atuar de forma significativa na promoção da saúde de adolescentes, uma vez que advém de conhecimentos capazes de serem utilizados na realização de busca ativa e identificação dos problemas enfrentados, corroborando para métodos de intervenção eficazes pautados por meio de ações educativas e utilização de recursos pedagógicos para prevenção à gravidez precoce e a utilização de métodos contraceptivos (Teles et al., 2014).

Desta forma, nota-se que dentre os desafios que o(a) enfermeiro(a), enquanto educador(a) em saúde, enfrenta para a redução das taxas de gravidez e Infecções Sexualmente Transmissíveis/AIDS entre os adolescentes, tem-se o empoderamento desses sujeitos ao comportamento sexual seguro.

Uma limitação deste estudo é de que os juízes apresentam bom nível de escolaridade (graduados e/ou pós-graduado) o que facilita a realização da tarefa de leitura e compreensão do conteúdo da história em quadrinhos. Além disso, o número de professores da educação básica participante do estudo foi menor do que o de profissionais de saúde e pesquisadores. Esses fatos sugerem a necessidade de estudos posteriores com os estudantes da educação básica, para verificar a aplicabilidade da mesma. Apesar disso, a história em quadrinhos se apresenta como instrumento válido e importante para facilitar o processo de ensino aprendizagem sobre métodos contraceptivos entre os adolescentes. 


\section{Conclusão}

Ao final do estudo, os resultados permitiram concluir que o objetivo proposto foi alcançado, uma vez que a História em Quadrinhos intitulada “Métodos Contraceptivos: Sou Jovem e ainda não quero gerar uma vida!”, obteve validade, pelos juízes, conferindo credibilidade de conteúdo e aparência.

Assim, evidencia-se que a HQ, aqui apresentada, é uma importante ferramenta para promover saúde, desenvolver habilidades e favorecer educação em saúde, principalmente ao público adolescente, haja vista ter sido validade por especialistas da área.

De tal modo que o presente estudo evidenciou a importância do desenvolvimento de recursos pedagógicos, que possam ser aplicáveis no processo de ensino aprendizagem aperfeiçoando e possibilitando a construção do conhecimento de forma interativa, facilitando a transmissão deste conhecimento por meio de mensagens gráficas e visuais. Além disso, proporcionou um instrumento que facilita o desenvolvimento da função do enfermeiro como educador em saúde, que necessita utilizar a comunicação verbal e escrita para o processo de cuidar, na perspectiva de alcançar a excelência do processo educativo em saúde.

Nesse sentido, torna-se relevante a contribuição deste estudo no contexto da educação em saúde e o papel desse recurso para se promover saúde, desenvolver habilidades e favorecer a autonomia e confiança do adolescente. Além disso é uma forma de fortalecer a prática educativa da enfermagem e, assim, minimizar os desafios enfrentados por não ter acesso aos avanços exigidos no processo ensino aprendizagem.

Dado o caráter deste estudo e o resultado alcançado, se faz necessário estudos posteriores para continuar o processo de validação para avaliação da eficiência da HQ construída e sua aplicabilidade junto aos estudantes da educação básica. Acredita-se que os seguintes pontos podem ser abordados em futuras pesquisas: validar a HQ junto aos estudantes da educação básica como facilitador ou como um auxílio para um aprendizado sobre a temática abordada; Realizar pesquisa-ação sobre o processo de ensino aprendizagem da HQ.

\section{Referências}

Amaral, L. C. Z., \& Locatelli, A. (2019). Produção de HQs como instrumento de avaliação de uma intervenção didática para ensino de reações químicas. Ensino de Ciências e Tecnologia em Revista, 9(1), 15-30. 10.31512/encitec.v9i1.2060

Beserra, E. P., Sousa, L. B., Cardoso, V. P., \& Alves, M. D. S. Perception of adolescents about the life activity "express sexuality". Revista Online de Pesquisa Cuidado é Fundamental, 9(2), 340-6. http:// dx.doi.org/10.9789/2175-5361.2017.v9i2.340-346

Costa, G. M., Figueredo, R. C., \& Ribeiro, M. S. (2013). A importância do enfermeiro junto ao PSE nas ações de educação em saúde em uma escola municipal de Gurupi, TO. Revista Científica do ITPAC, 6(2). Retrieved from: https://assets.itpac.br/arquivos/Revista/62/6.pdf

Dias, P. M. M., Oliveira, J. M., Lustosa, A. P., Lima, H. K. L. S., Moreira, K. A. P., \& Pereira, T. M. (2017). Repercussions of teenage pregnancy in the life of adult women. Revista Rene, 18(1), 106-13. http://dx.doi.org/ 10.15253/2175-6783.2017000100015

Finotti, M. (2015). Manual de anticoncepção. São Paulo: Federação Brasileira das Associações de Ginecologia e Obstetrícia. https://armazemdaciencia.files.wordpress.com/2017/08/manual-anticoncepcao-febrasgo-2015-pdf.pdf

Haynes, S. N., Richard, D. C. S., \& Kubany, E. S. (1995). Content validity in psychological assessment: A functional approach to concepts and methods. Psychological Assessment, 7(3), 238-247. https://doi.org/10.1037/1040-3590.7.3.238

Lacerda, T. T. B., Magalhães, L. de C., \& Rezende, M. B. (2007). Validade de conteúdo de questionários. Revista de Terapia Ocupacional da Universidade de São Paulo, 18(2), 63-77. https://doi.org/10.11606/issn.2238-6149.v18i2p63-77

Lei Federal n. 8069, de 13 de julho de 1990. Dispõe sobre o Estatuto da Criança e do Adolescente e dá outras providências. http://www.planalto.gov.br/ccivil_03/leis/18069.htm

Ministério da Saúde. (2015). Protocolo clínico e diretrizes terapêuticas para atenção integral às pessoas com infecções sexualmente transmissíveis. Secretaria de Vigilância em Saúde. Departamento de DST, Aids e Hepatites Virais. Brasília: Ministério da Saúde. 
Research, Society and Development, v.10, n.1, e2010111214, 2021 (CC BY 4.0) | ISSN 2525-3409 | DOI: http://dx.doi.org/10.33448/rsd-v10i1.11214

Ministério da Saúde. (2016). Protocolos da Atenção Básica: Saúde das Mulheres. Instituto Sírio-Libanês de Ensino e Pesquisa. Brasília: Ministério da Saúde, 2016. http://bvsms.saude.gov.br/bvs/publicacoes/protocolos_atencao_basica_sau de_mulheres.pdf

Oviedo, H., \& Campo-Arias, A. (2005). Aproximación al uso del coeficiente alfa de Cronbach. Revista Colombiana de Psiquiatría, 34(4). https://www.redalyc.org/articulo.oa?id=80634409

Paranhos, R., Figueiredo, D. B., F., Rocha, E. C., Silva, J. A., Jr., \& Freitas, D. (2016). Uma introdução aos métodos mistos. Sociologias, 18(42), 384-411. https://doi.org/10.1590/15174522-018004221

Pedreira, R. B. S., Rocha, S. V., Santos, C. A., Vasconcelos, L. R. C., \& Reis, M. C. (2016). Content validity of the Geriatric Health Assessment Instrument. Einstein, 14(2), 158-77. http://dx.doi.org/10.1590/S1679-45082016AO3455

Polit, D. F., Beck, C. T., \& Hungler, B. P. (2011). Fundamentos de pesquisa em enfermagem: métodos, avaliação e utilização (6a ed.). Porto Alegre: Artmed.

Presser, A. T. R., \& Schlögl, L. (2013). Histórias em quadrinhos enquanto meio de comunicação eficaz. Razón Palabra, 17(83), 259-75. http://www.revistarazonypalabra.org/index.php/ryp/article/view/530/559

Rama, A., \&Vergueiro, W. (Orgs.). (2014). Como usar histórias em quadrinhos na sala de aula. (4a ed). São Paulo: Contexto.

Ribeiro, V. C. S., Nogueira, D. L., Assunção, R. S., Silva, F. M. R., \& Quadros, K. A. N. (2016). Papel do enfermeiro da estratégia de saúde da família na prevenção da gravidez na adolescência. Revista de Enfermagem do Centro Oeste Mineiro, 1(6), 195775. http://dx.doi.org/10.19175/recom.v0i0.881

Sales, J. K. D. de, Sales, J. K. D. de, Alves, D. de A., Coelho, H .P., Oliveira, O. P. de, \& Santos, R. L. dos. (2020). Fatores de risco associados ao comportamento sexual de adolescentes. Revista Eletrônica Acervo Saúde, 49:e3382. https://doi.org/10.25248/reas.e3382.2020

Santos, T. C., \& Pereira, E. G. C. (2013). Histórias em quadrinhos como recurso pedagógico. Revista Práxis, 5(9), 51-6. https://sites.unifoa.edu.br/praxis/numeros/09/51-56.pdf

Silva, M. R. B., Silva, L. A., Maturana, H. C. A., Silva, R. B., Santos, M. E., \& Figueiredo Filho, V. (2015). Porque elas não usam? um estudo sobre a não adesão das adolescentes ao preservativo e suas repercussões. Revista Saúde em Redes, 1(4), 75-83. http://dx.doi.org/10.18310/2446-4813.2015v1n4p75-83

Silva, Q. A. D., Menegon, V. G. S., Nunes, M. A. S., \& Silva, R. R. (2018). Gravidez na adolescência: a percepção de parturientes adolescentes. Revista Ciência \& Saberes, 4(3), 1152-1157. http://www.facema.edu.br/ojs/in dex.php/ReOnFacema/article/view/304

Teles, L. M. R., Oliveira, A. S., Campos, F. C., Lima, T. M., Costa, C. C., Gomes, L. F. S., Oriá, M. O. B., et al. (2014). Development and validating an educational booklet for childbirth companions. Revista da Escola de Enfermagem da USP, 48(6), 977-84. http://dx.doi.org/10.1590/S0080-623420140000700003

Tibúrcio, M. P., Melo, G. S. M., Balduíno, L. S. C., Costa, I. K. F., Dias, T. Y. A. F., \& Torres, G. V. (2014). Validação de instrumento para avaliação da habilidade para mensuração da pressão arterial. Revista Brasileira de Enfermagem, 67(4), 581-7. http://dx.doi.org/10.1590/0034-7167.2014670413 